\title{
Generalized peritonitis after spontaneous rupture of pyonephrosis: a case report
}

This article was published in the following Dove Press journal: International Medical Case Reports Journal

\author{
Desalegn Markos Shifti' \\ Kebebe Bekele ${ }^{2}$ \\ 'St. Paul Hospital Millennium Medical \\ College, Addis Ababa, Ethiopia; \\ ${ }^{2}$ Department of Surgery, School of \\ Medicine, Madda Walabu University, \\ Bale Goba, Ethiopia
}

Background: Peritoneal fistulization of a pyonephrosis is an extremely rare event which invariably leads to generalized peritonitis. This is a very rare case report on generalized peritonitis after spontaneous rupture of pyonephrosis.

Case presentation: A 28-year-old male patient from the rural part of Bale zone, Ethiopia, was admitted to Goba Referral Hospital with high-grade fever, diffused abdominal pain and abdominal distension. Initially, he experienced colicky and intermittent pain that made him stay at home for 2-3 days. He then started to develop constant left flank pain which gradually got worse and was associated with urinary frequency of approximately 5-6 times/day, high-grade intermittent fever, chills, rigors and loss of appetite. With the diagnosis of generalized peritonitis, we resuscitated him with two bags of normal saline and one bag of ringer lactate intravenously. During an abdominal ultrasound examination we identified that the left kidney was replaced by an abscess containing sac, and there was a huge intraperitoneal loculated abscess with internal septation and an associated free inter-loop and pelvic echo debris abscess. When we performed an exploratory laparotomy, 1 L-thick abscess from the general peritoneum was aspirated and early fibrinous inter-loop adhesion was identified. In addition, there was a large retroperitoneal cystic abscess containing sac extended from the spleen up to the pelvic brim crossing the midline to the right side and bulged intraperitoneally. Furthermore, a $1.5 \mathrm{~cm}$ wide perforation that pour abscess in to peritoneal cavity was found. A total of $4 \mathrm{~L}$ of puss was removed from the left kidney. As treatment, since the left kidney lost all function and became a pus-contacting sac, we performed a left-sided nephrectomy and abdominal lavage. Postoperatively, the patient had an uneventful recovery and was discharged from the hospital on the eighth day. We followed him for 6 months, and kidney function tests were normal and he did not develop any complications. Conclusion: This case report highlighted the importance of recognizing the possibility of underlying kidney rupture in a patient with generalized peritonitis. Uretero-pelvic junction obstruction (UPJO) might be the possible cause of pyonephrosis in our case. As a treatment, nephrectomy is a preferable option when the affected kidney is not fully functional and the contralateral kidney is normal.

Keywords: pyonephrosis, generalized peritonitis, abscess, perforation, nephrectomy, case report

\section{Introduction}

The accumulation of purulent exudate in the hydronephrotic collecting system and abscess formation constitute the pathophysiology of pyonephrosis. ${ }^{1}$ If it is not diagnosed early, it can worsen rapidly and cause the patient to die of septic shock. ${ }^{2}$ Peritoneal fistulization of a pyonephrosis is an extremely rare event which invariably leads to generalized peritonitis. $^{3}$
Correspondence: Desalegn Markos Shifti St. Paul Hospital Millennium Medical College, P.O. Box I27I, Addis Ababa 1000, Ethiopia

Tel +25I 9l 2057720

Email desalegnmarkos@gmail.com 
The risk factors for pyonephrosis include an immunosuppressive status and an anatomic urinary tract obstruction, of which $75 \%$ is nephrolithiasis related. ${ }^{4}$

Antibiotics have no effect in pyonephrosis unless the pus is surgically drained. Percutaneous nephrostomy and urethral catheter insertions are, therefore, necessary. In addition, studies showed that percutaneous drainage is a fast, trusted and effective diagnostic and therapeutic method..$^{5-7}$

Radical nephrectomy can be the preferred treatment for a kidney that has lost most of its function if the contralateral kidney is normal. Nephrectomy has been found to have fewer complications compared to other treatments. ${ }^{8,9}$ The aim of this paper was to present an unusual case of generalized peritonitis after spontaneous rupture of pyonephrosis diagnosed in a 28-year-old man who was admitted to Goba Referral Hospital with high-grade fever, diffused abdominal pain and abdominal distension.

\section{Case presentation}

A 28-year-old male patient from the rural part of Bale zone, Ethiopia, was admitted to Goba Referral Hospital with highgrade fever, diffused abdominal pain and abdominal distension. He is a farmer and married with four children. The patient had experienced left flank pain for the past 6 years. Initially, he experienced colicky and intermittent pain that made him stay at home for 2-3 days. In addition, he had a history of reddish discoloration of urine during the flank pain episode. At the beginning of this condition, he did not visit any health care institution. He then started to develop constant left flank pain which gradually got worse and was associated with urinary frequency of around 5-6 times/day, high-grade intermittent fever, chills, rigors and loss of appetite. After 3 weeks of the abovementioned symptoms, he was admitted to Ginnir hospital (one of the rural hospitals in Ethiopia) for 7 days with the diagnosis of left pyelonephritis and was given ceftriaxone $1 \mathrm{~g}$ intravenous (IV) bid and diclofenac $75 \mathrm{mg}$ intramuscular (im) and as needed (PRN). Despite this, after 6 days of stay in the aforementioned hospital, he developed generalized abdominal pain, abdominal distension, constipation and high-grade intermittent fever. Upon arrival to our hospital, Goba Referral Hospital, the patient was acutely sick looking and had a blood pressure (BP) of $80 / 50 \mathrm{mmHg}$, a pulse rate (PR) of 138 beats/minute, a respiratory rate (RR) of 34 breaths $/$ minute and a temperature of $38.9^{\circ} \mathrm{C}$. Besides, the patient had pink conjunctiva and dry tongue. He had clear chest on auscultation, and first heart sound or lub (S1) and second heart sound or dub (S2) were well heard when the heart was assessed. Regarding the abdominal assessment, his abdomen was distended, had no visible peristalsis, and it did not move with respiration. Also there was hypoactive bowel sound, guarding, rigidity, direct and rebound tenderness all over abdomen. The digital rectal examination showed empty rectum. He had a cold extremity. He had no history of diabetic mellitus, hypertension and he was neither a smoker nor an alcohol drinker. With the diagnosis of generalized peritonitis, we resuscitated him with two bags of normal saline and one bag of ringer lactate intravenously, causing his BP to became 100/60 mmHg. The laboratory investigation revealed the following: red blood cells, $201 \mathrm{~g} / \mathrm{dL}$; hemoglobin, $13.4 \mathrm{~g} / \mathrm{dL}$; white blood cells, 20,000/ $\mu \mathrm{L}$; granulocyte, $88 \%$; platelet counts, $236,000 / \mathrm{L}$; blood group, $\mathrm{A}^{+}$and HIV antibody test, negative. In abdominal ultrasound examination, we identified that the left kidney was replaced by an abscess-containing sac, and there was huge intraperitoneal loculated abscess with internal septation and associated free inter-loop and pelvic echo debris abscess. After 2 hours of IV fluid administration, he produced $80 \mathrm{~mL}$ of urine. When we performed an exploratory laparotomy, 1 L-thick abscess from general peritoneum was aspirated, and early fibrinous inter-loop adhesion was identified. In addition, there was a huge retroperitoneal cystic abscess-containing sac extended from the spleen up to the pelvic brim crossing the midline to the right side and bulged intraperitoneally. Furthermore, about a $1.5 \mathrm{~cm}$ wide perforation that pour abscess in to peritoneal cavity was found. A total of $4 \mathrm{~L}$ of puss were removed out from the left kidney. As treatment, since the left kidney had lost full function (Figure 1) and became a pus-contacting sac, while the contralateral kidney was still normal, we performed a left-sided nephrectomy and abdominal lavage. When we mobilized the left colon during the intraoperative phase, adhesion and renal pedicle were identified. Therefore, double ligation of renal artery and vein together was done with silk number 1 as a result of inability to separate the renal artery and vein due to dense adhesion. Then, the ureter was ligated and divided. The abdominal wall was closed after the lavage drain was left inside. We did not find any stones in the affected kidney and nearby structure. Moreover, there was no evidence of tumor and tuberculosis from histopathologic examination of resected sample. Therefore, uretero-pelvic junction obstruction (UPJO) might be the possible cause of pyonephrosis of this case. Postoperatively, we put the patient on ceftriaxone $1 \mathrm{~g}$ IV bid, metronidazole $500 \mathrm{mg}$ IV tid, maintenance fluid, tramadol $50 \mathrm{mg}$ IV qid and diclofenac $50 \mathrm{mg}$ im tid. He started sips on the third postoperative day. We removed the drain on the fifth postoperative day. The renal function test (RFT) performed in postoperative phase revealed normal range of serum electrolyte, and there was a normal amount of urine output. The patient had an uneventful recovery and was 


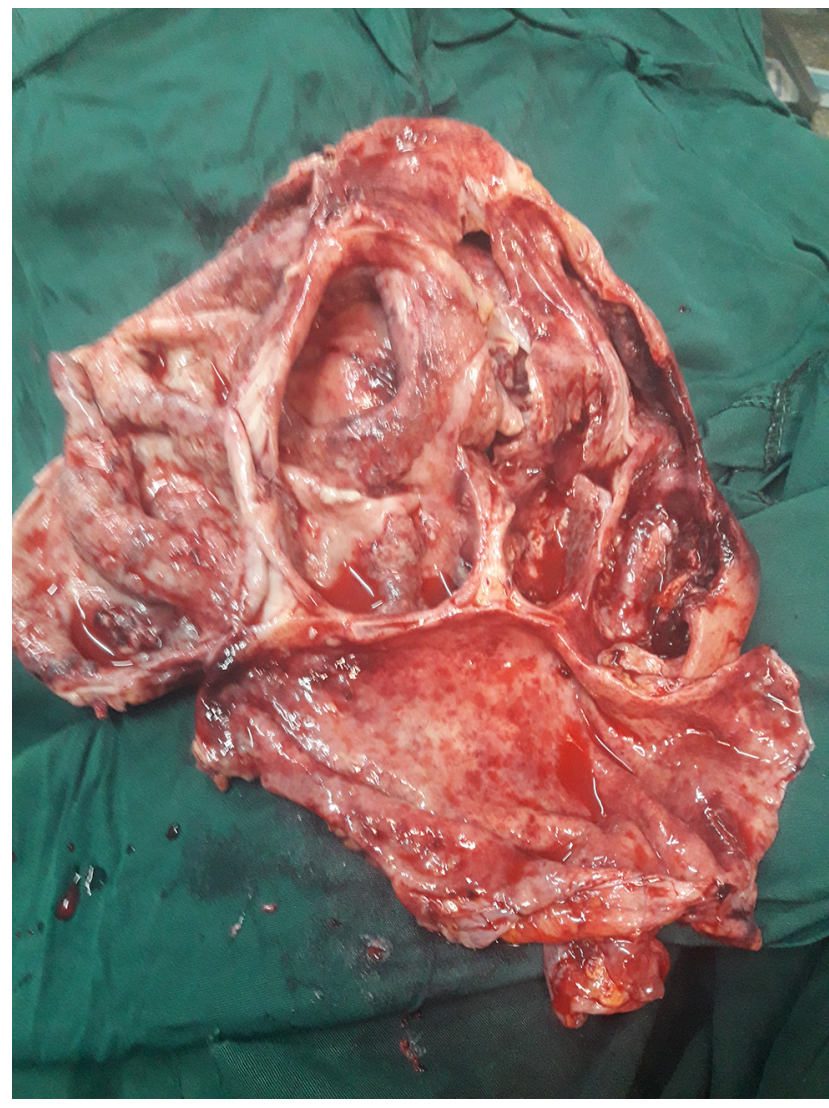

Figure I Macroscopic view of the left kidney after nephrectomy was performed.

discharged from the hospital on the eighth day after surgery. We followed him for 6 months, and he had a normal kidney function test and did not develop any complications.

\section{Consent}

Written informed consent was obtained from the patient for publication of this case report.

\section{Discussion}

The initial symptom of our patient was left flank pain which gradually got worse and was associated with urinary frequency of around 5-6 times/day, high-grade intermittent fever, chills, rigors and loss of appetite. Literature also supported that the most common symptoms of pyonephrosis are fever, chills and flank pain. ${ }^{10-14}$

We did not find any stones in the affected kidney, and UPJO might be the possible cause of pyonephrosis in our case. On the other hand, different authors reported that $75 \%$ of pyonephrosis cases are due to nephrolithiasis, ${ }^{4} 71 \%$ of the cases are due to urinary lithiasis ${ }^{15}$ and 17 out of 23 cases are due to nephrolithiasis. ${ }^{6}$ This may imply that pyonephrosis is a multifactorial disease, and a further study should be conducted to clearly present its risk factors and causes.
Our diagnosis was based on ultrasound. Different literature also confirmed the importance and even the indispensability of ultrasound and computed tomography in the diagnosis of pyonephrosis. ${ }^{2,10,12,13,16,17}$ The sensitivity of renal ultrasonography for differentiating hydronephrosis from pyonephrosis is $90 \%$, and the specificity is $97 \% .{ }^{17}$

The ultrasound finding in this patient indicated that the left kidney was replaced by an abscess-containing sac and a huge intraperitoneal loculated abscess with internal septation, associated free inter-loop and pelvic echo debris abscess. Literature also supported that pyonephrosis might be differentiated from hydronephrosis on ultrasonography by the presence of debris, fluid-fluid levels and internal echoes in the collecting system. ${ }^{18}$

To treat the patient, left-sided nephrectomy and abdominal lavage were performed. The patient had no postoperative complications. Literature also supported that radical nephrectomy can be the preferred treatment for a kidney that has lost most of its function if the contralateral kidney is normal. On top of this, nephrectomy has been found to have fewer complications compared to other treatments. ${ }^{9,19}$

\section{Conclusion}

This case report highlights the importance of recognizing the possibility of underlying kidney rupture in a patient with generalized peritonitis. UPJO might be the possible cause of pyonephrosis in our case. Regarding its treatment, nephrectomy is a preferable option when the affected kidney is not fully functional and the contralateral kidney is normal.

\section{Disclosure}

The authors report no conflicts of interest in this work.

\section{References}

1. Eroğlu M, Kandıralı E. Akut Pyelonefrit ve pyonefroz. Turk Klin J Surg Med Sci. 2007;3(20):24-28.

2. Erol A, Çoban S, Tekin A. A giant case of pyonephrosis resulting from nephrolithiasis. Case Rep Urol. 2014;2014:1-3.

3. Balas $P$, Seqditsas T, Antonopoulos D. Peritonitis after spontaneous rupture of pyonephrotic kidney into a peritoneal cavity. Am J Surg. 1971;121(5): 612-613.

4. Chen YL, Wang MC, Huang JZ. Pyonephrosis. Kidney Dial. 2008;20: 14-18.

5. Eroğlu M, Kandıral E. Akut Pyelonefrit ve pyonefroz. Turk Klin J Surg Med Sci. 2007;3(20):24-28.

6. Lezin MS, Hofmann R, Stoller ML. Pyonephrosis: diagnosis and treatment. Br J Urol. 1992;70(4):360-363.

7. Mokhmalji H, Braun PM, Portillo FJM. Percutaneous nephrostomy versus ureteral stents for diversion of hydronephrosis caused by stones: a prospective, randomized clinical trial. J Urol. 2001;165(4):1088-1092.

8. Jimenez JF, Pacios MAL, Llamazares G, Conejero J, Sole-Balcells F. Treatment of pyonephrosis: a comparative study. J Urol. 1978;120(3) $287-289$. 
9. Androulakakis PA. Pyonephrosis: a critical review of 131 cases. $B r J$ Urol. 1982;54(2):89-92.

10. Scarneciu I, Constantina A, Grigorescu D, Maxim L. Pyonephrosis: diagnosis and treatment: report of 65 cases. J Mol Biol. 2015;2:122-125.

11. Taheri AA, El Aziz S, Chadli A. Acute pyelonephritis in diabetic patients. Ann D'Endocrinol. 2015;76(4):558.

12. Sow Y, Fall B, Sarr A, Thiam A, Diao B, Fall PA. Pyonephrosis: 44 cases in senegal. Med Trop (Mars). 2011;71(5):495-498.

13. Mohamed A, Mohammed R. Management of pyonephrosis: our experience. Webmed Cent Urol. 2012;3(5):WMC003420.

14. Hasigov A, Engbang JPN, Fidarov F. Giant pyonephrosis due to urolithiasis and diabetes: case report. Open J Urol. 2016;6(7):122-125.

15. Rabii R, Joual A, Raisetal H. Pyonephrosis: diagnosis and treatment: a review of 14 cases. Ann D'Urol. 2000;34(3):161-164.
16. Schaeffer AJ, Schaeffer EM. Infections of the urinary tract. In: Wein AJ, Kavoussi LR, editors. Campbell-Walsh Urol. 9th ed. Philadelphia, PA: Saunders Elsevier; 2007:1973-1985.

17. Van Nieuwkoop C, Hoppe BP, Bonten TN, et al. Predicting the need for radiologic imaging in adults with febrile urinary tract infection. Clin Infect Dis. 2010;51(11):1266-1272.

18. Schaeffer AJ, Schaeffer EM. Infected hydronephrosis and pyonephrosis. In: Wein AJ, Kavoussi LR, Novick AC, Partin AW, Peters CA, editors. Campbell-Walsh Urology. 10 ed. Philadelphia, PA: Saunders Elsevier; 2012:3560-3574.

19. Jimenez JF, Lopez Pacios MA, Llamazares G, Conejero J, SoleBalcells F. Treatment of pyonephrosis: a comparative study. J Urol. 1978;120(3):287-289.

\section{Publish your work in this journal}

The International Medical Case Reports Journal is an international, peer-reviewed open-access journal publishing original case reports from all medical specialties. Previously unpublished medical posters are also accepted relating to any area of clinical or preclinical science. Submissions should not normally exceed 2,000 words or
4 published pages including figures, diagrams and references. The manuscript management system is completely online and includes a very quick and fair peer-review system, which is all easy to use. Visit $\mathrm{http}$ ///www.dovepress.com/testimonials.php to read real quotes from published authors.

Submit your manuscript here: https://www.dovepress.com/international-medical-case-reports-journal-journal 